\title{
Los Factores de Protección frente a la Conducta Antisocial: ¿Explican las diferencias en violencia entre chicas y chicos?
}

\author{
Raquel Bartolomé (*), Marta Montañés, Cristina Rechea \& Juan Montañés \\ Universidad de Castilla - La Mancha \\ (*):Raquel.Bartolome@uclm.es
}

Original recibido el 5 de marzo de 2009

\section{RESUMEN}

La investigación sobre conducta antisocial y violenta en adolescentes muestra de forma persistente que los chicos conforman el grupo de riesgo para desarrollar una carrera delictiva seria y, especialmente, violenta. Dos posibles hipótesis se han planteado para explicar este hecho: a) que chicas y chicos estén diferencialmente expuestos a los mismos factores de riesgo/protección y b) que el efecto de esos factores sea diferente en cada grupo. Este trabajo tiene como objetivos explorar las semejanzas y diferencias de comportamiento entre chicas y chicos adolescentes y poner a prueba las dos explicaciones señaladas con respecto a diversos factores de protección considerados relevantes en la literatura. Para ello se utilizan los datos recabados a través de autoinforme entre 642 adolescentes escolarizados de Albacete. Los resultados muestran que, aunque las chicas están más expuestas a ciertos factores de protección, esto no explica por completo su menor tendencia a la violencia. Por otro lado, al contrario de lo esperado, el efecto protector de los factores parece ser mayor en los chicos. Se discuten estos resultados y sus implicaciones.

Palabras clave: conducta antisocial, adolescencia, diferencias, factores protectores, autoinforme, estudio ex post facto.

\begin{abstract}
Nowadays, boys and girls in Spain show similar patterns of antisocial behaviour. Nevertheless, data indicates that differences in violent behaviour continue being important. Two hypotheses try to explain the difference: a) girls might be more exposed to certain factors of protection, or, b) factors' protective effects are higher for girls. Using self-reports data, collected in 2004 among 649 teenagers from three Schools in Albacete (Spain), the current study examines these hypotheses. The self-report questionnaire includes items on antisocial behaviour and risk/protective factors adapted from the ISRD-I and from the California Healthy Kids Survey (2002). The results provide partial support for the differential exposition hypotheses but contradictory evidence regarding the differential protective effects. The implications of these findings for future investigations are discussed.
\end{abstract}

Key words: antisocial behaviour, adolescence, differences, protective factors, selfreport. 


\section{Introducción}

Existe en la actualidad un enorme interés por mejorar el conocimiento sobre la conducta antisocial de adolescentes y jóvenes; lógicamente, se espera que de tal conocimiento se deriven intervenciones preventivas y tratamientos más exitosos del problema. En este ámbito, una de las líneas de investigación con más desarrollo es la dedicada a identificar los factores de riesgo y protección frente a la conducta antisocial y violenta. De hecho, se han conseguido identificar múltiples factores de riesgo y se está trabajando con ahínco en detectar también factores protectores (Lipsey y Derzon, 1998; Lösel y Bender, 2003; Reppucci, Fried y Schmidt, 2002). Sin embargo, en los estudios sobre factores de riesgo/protección se observa todavía una carencia tradicional en la investigación sobre delincuencia: apenas se presta atención al papel del sexo/género (Belknap, 1996; Pollock, 1999).

Es bien conocido que el sexo constituye, junto con la edad, el principal correlato de la conducta antisocial y violenta, de tal forma que los varones, sobre todo los varones jóvenes, están sobre representados en todas las estadísticas sobre delincuencia. Y esto es así en todo el mundo, independientemente de que los datos sean de auto-informe $\mathrm{u}$ oficiales (Rutter, Giller y Hagell, 2000). Esto plantea dos cuestiones importantes: ¿por qué los chicos delinquen más que las chicas?, pero también, ¿por qué delinquen las chicas que lo hacen?, lo que en la criminología anglosajona se conoce como "the gender gap": la cuestión sexo/género. Generalmente, los investigadores sociales han mostrado poco interés en estudiar estas cuestiones. En este sentido, se considera que la mayoría de las teorías e investigaciones sobre "delincuencia juvenil" son, en realidad, teorías sobre la delincuencia juvenil masculina, que sólo recientemente se están matizando para dar cuenta de las diferencias entre chicas y chicos. Este estado de cosas coloca a las chicas antisociales y delincuentes en una clara situación de desventaja, ya que los programas de prevención y/o intervención no se han diseñado atendiendo a sus características y necesidades, sino a la de los chicos (Belknap y Holsinger, 1998; Chesney-Lind, 1997).

Dos hipótesis han tratado de explicar la mayor tendencia de los chicos a la conducta antisocial seria y violenta. La primera que existe una exposición diferencial de chicos y chicas a los mismos factores de riesgo/protección. Fundamentalmente, se ha hecho referencia a la socialización diferencial. Así, se señala que las chicas tienen más 
vínculos pro sociales con la escuela y los amigos y están más supervisadas por los padres, factores todos ellos que sirven para proteger frente a la conducta antisocial de acuerdo con las teorías del control social (Chapple, McQuillan, y Berdahl, 2005).

Otra hipótesis plantea, en cambio, que las variables de riesgo/protección tienen efectos diferentes en chicos y chicas y que el sexo/género no es una variable más, sino que afecta sustancialmente al impacto que las experiencias, sucesos, etc. tienen sobre los individuos (Heimer y De Coster, 1999; Steffensmeier y Allan, 1996), por ejemplo los chicos pueden verse más influidos que las chicas por sus amigos antisociales.

En nuestra opinión, conocer mejor estas cuestiones puede ser clave para entender la conducta antisocial y violenta de los jóvenes e intervenir sobre ella adecuadamente. Por ello, nos planteamos tres objetivos fundamentales en este trabajo: a) comprobar las diferencias y semejanzas entre chicos y chicas en sus patrones de conducta antisocial, b) analizar si existe una exposición diferencial de chicos y chicas a los mismos factores de protección y c) estudiar si los efectos de las variables de protección identificadas tienen efectos diferentes en chicos y chicas.

\section{Método}

\section{Participantes}

La muestra está compuesta por 642 estudiantes de Educación Secundaria Obligatoria y Post-obligatoria de tres centros de la provincia de Albacete, de los cuales $319(49,7 \%)$ son chicos y $323(50,3 \%)$ son chicas, con edades entre los 12 y los 21 años (media de edad: 15,20 años).

La muestra fue seleccionada por conveniencia, pero se cumplieron cuotas de muestreo por edades y sexo. Además, se seleccionaron centros de zonas con características socioeconómicas distintas, dos de ellos en el ámbito urbano y uno en el ámbito rural.

\section{Instrumento}

El instrumento utilizado fue un cuestionario auto-aplicado denominado Encuesta sobre Estilos de Vida de los Adolescentes, diseñado por el Centro de Investigación en Criminología de la UCLM (Albacete). Para su diseño, se han utilizado ítems del ISRD I en lo referido a conductas antisociales y violentas (Rechea, Barberet y Montañés, 1995), 
y de la Encuesta a los Muchachos y Muchachas Saludables de California (California Healthy Kids Survey, 2002), en especial, de la parte dedicada a la resiliencia (RYDM) y a factores de protección. Los ítems que miden protección y resiliencia en el CHKS fueron seleccionados por su relevancia en la literatura y elaborados por expertos en este tema (Bernard, 1999; Constantine, Bernard y Díaz, 1999).

Los factores de protección y resiliencia incluidos son: Factores protectores

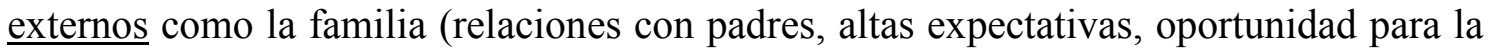
participación significativa y supervisión), la escuela (alta vinculación escolar, participación significativa y percepción de trato justo por los profesores) y los amigos (nivel alto de apoyo, amigos pro sociales). Factores protectores internos: apoyo social en la resolución de problemas, empatía, auto-eficacia, autoconocimiento, objetivos y actitudes hacia el futuro, resolución pacífica de los problemas. La agrupación de los ítems en estos factores se ha comprobado a través de un análisis factorial varimax.

\section{Procedimiento}

Los datos fueron recogidos en marzo y abril de 2004. El cuestionario se administró en grupo, en horario de tutoría para no interferir en el curso normal de las clases. Se pidió a los adolescentes que contestasen de forma individual y sincera, asegurándoles el anonimato y la confidencialidad de los datos.

\section{Resultados}

En relación con nuestro primer objetivo, hemos llevado a cabo análisis de la prevalencia, la frecuencia y la variedad de las conductas antisociales cometidas por chicos y chicos. Los datos de prevalencia muestran que el porcentaje de chicas y chicos que ha cometido las conductas estudiadas es similar en ambos grupos. En el caso de consumo de drogas legales es incluso ligeramente superior en las chicas. El porcentaje de chicos implicados es significativamente superior en las conductas de haber sido expulsado del centro escolar, vandalismo, llevar armas, participar en peleas y comprar algo robado (todas ellas realizadas “alguna vez"). En general, pues, la diferencia fundamental es que más chicos que chicas han llevado a cabo alguna conducta violenta en su vida. 
Para conocer si existen diferencias en la frecuencia con que chicos y chicas realizan las diferentes conductas se ha realizado una prueba $t$; los resultados muestran que la incidencia en las conductas antisociales en el último año es semejante en ambos grupos, aunque ligeramente mayor en los chicos. Sólo aparecen frecuencias significativamente mayores en los chicos en el número de expulsiones del centro educativo $(2,39$ vs. 1,$23 ; \mathrm{t}=2,267, \mathrm{p}=, 03)$ y en haberse emborrachado $(2,73$ vs. 1,$90 ; \mathrm{t}=$ $2,820, \mathrm{p}=, 000)$.

\begin{tabular}{|c|c|c|c|}
\hline Conductas antisociales "alguna vez" & Chicos & Chicas & $\chi^{2}$ (g.l.) \\
\hline \multicolumn{4}{|l|}{ Consumo de drogas } \\
\hline Consumir tabaco & $52,2 \%$ & $60,2 \%$ & $4,022(1)^{*}$ \\
\hline Consumir alcohol & $66,6 \%$ & $67,4 \%$ & N.S. \\
\hline Consumir otras drogas & $25,2 \%$ & $23,5 \%$ & N.S. \\
\hline \multicolumn{4}{|l|}{ Conductas anti-normativas } \\
\hline Faltar al colegio & $46,4 \%$ & $51,4 \%$ & N.S. \\
\hline Escaparte de casa & $10,0 \%$ & $9,3 \%$ & N.S. \\
\hline Irse/colarse sin pagar & $26,0 \%$ & $21,7 \%$ & N.S. \\
\hline Robar algo & $24,8 \%$ & $23,5 \%$ & N.S. \\
\hline Comprar algo robado & $11,9 \%$ & $6,2 \%$ & $6,390(1)^{*}$ \\
\hline Vender drogas & $1,9 \%$ & $2,5 \%$ & N.S. \\
\hline Expulsión del centro educativo & $12,1 \%$ & $6,3 \%$ & $6,404(1)^{* *}$ \\
\hline \multicolumn{4}{|l|}{ Conductas violentas } \\
\hline Vandalismo & $20,0 \%$ & $10,6 \%$ & $10,775(1)^{* *}$ \\
\hline Discusión fuerte & $52,5 \%$ & $50,8 \%$ & N.S. \\
\hline Llevar armas & $22,2 \%$ & $6,5 \%$ & $32,229(1)^{* *}$ \\
\hline Amenazar con agredir físicamente & $29,8 \%$ & $22,3 \%$ & N.S. \\
\hline Peleas & $49,0 \%$ & $27,1 \%$ & $31,590(1)^{* *}$ \\
\hline
\end{tabular}


Para conocer la variedad de conductas que ha realizado cada uno de los sujetos de la muestra, se han hallado tres índices: índice de violencia (número de conductas violentas distintas en las que ha participado); índice de conducta anti-normativa (número de conductas anti-normativas diversas que ha llevado a cabo) e índice de consumo (número de sustancias distintas consumidas). Como ocurría al analizar la prevalencia, no aparecen diferencias significativas en la variedad de conductas antisociales cometidas, ni de sustancias consumidas, pero sí en la variedad de conductas violentas, presentando los chicos una media mayor que las chicas $(1,68$ vs. 1,$15 ; \mathrm{t}=$ $4,750, \mathrm{p}=, 000)$.

Los datos nos indican, por tanto, que hay grandes semejanzas entre chicas y chicos en su conducta antisocial; las únicas diferencias se refieren a la participación en conductas violentas: más chicos han sido violentos $\mathrm{y}$, además, han cometido una mayor variedad de conductas violentas distintas.

La segunda parte de este trabajo se centra en analizar posibles explicaciones de esta diferencia entre chicos y chicas en conducta violenta. Para ello hemos analizado si las chicas están más expuestas que los chicos a las variables protectoras frente a la violencia. Previamente se comprobó que los factores estudiados tenían relación con la violencia. Se pudo comprobar que casi todos los factores de protección estudiados tenían una correlación significativa con el índice de violencia, excepto el apoyo percibido de los amigos. Los factores que muestran una relación más fuerte son: resolución pacífica de problemas $(-, 421, p=, 000)$, tener amigos pro sociales $(-, 336, p=$ $.000)$, la supervisión familiar $(-, 261, \mathrm{p}=, 000)$ y la relación con la madre $(-, 261, \mathrm{p}=, 000)$.

Los datos sobre exposición diferencial muestran que las chicas tienen una mayor supervisión paterna, más interés en seguir estudiando y su estilo de resolver problemas se centra más en la comunicación y es más pacífico. Además, tienen más relaciones con amigos pro sociales, que hacen lo correcto. Es decir, las chicas están más expuestas que los chicos a un número relevante de factores de protección, fundamentalmente, factores relacionados con la teoría del control social, como una mayor supervisión familiar y más vínculos con amigos pro sociales. Sin embargo, aunque muestran un mayor interés por seguir estudiando, su vinculación con el centro escolar es, en general, similar al de los chicos. 


\begin{tabular}{|c|c|c|c|c|c|}
\hline \multirow[b]{2}{*}{ Factores Protectores } & \multicolumn{2}{|c|}{ Chicos } & \multicolumn{2}{|c|}{ Chicas } & \multirow[t]{2}{*}{ t (gl) } \\
\hline & Media & D.T. & Media & D.T. & \\
\hline \multirow[t]{2}{*}{ Vinculo escolar } & 3,63 & ,81 & 3,85 & 80 & $-3,40$ \\
\hline & & & & & $(628)^{* *}$ \\
\hline Participación significativa en la escuela & 3,31 & 1,13 & 3,95 & ,96 & - \\
\hline Trato justo de los profesores & 3,26 & 1,09 & 3,40 & ,93 & - \\
\hline Participación significativa en casa & 3,46 &, 81 & 3,49 & 83 & - \\
\hline Expectativas familiares & 4,51 & 60 & 4,57 &, 56 & - \\
\hline \multirow[t]{2}{*}{ Supervisión familiar } & 3,61 & 1,13 & 3,95 & ,96 & $-4,07$ \\
\hline & & & & & $(600)^{* *}$ \\
\hline Relación con la madre & 4,38 &, 79 & 4,43 & ,75 & - \\
\hline Relación con el padre & 4,26 &, 89 & 4,19 & 9,36 & - \\
\hline \multirow[t]{2}{*}{ Apoyo de amigos } & 3,82 & 1,06 & 4,51 &, 72 & $-9,57$ \\
\hline & & & & & $(633)^{* *}$ \\
\hline \multirow[t]{2}{*}{ Amigos prosociales } & 3,46 & ,77 & 3,85 & 80 & $-5,95$ \\
\hline & & & & & $(622)^{* *}$ \\
\hline \multirow[t]{2}{*}{ Empatía } & 3,93 &, 81 & 4,31 &, 57 & $-6,84$ \\
\hline & & & & & $(630)^{* *}$ \\
\hline Autoeficacia & 4,03 & 69 & 3,91 & 61 & $2,19(616)^{* *}$ \\
\hline Autoconocimiento & 4,01 &, 88 & 3,87 & 88 & - \\
\hline Objetivos de futuro & 4,11 & 83 & 4,25 & 84 & $-2,02(627)^{*}$ \\
\hline \multirow[t]{2}{*}{ Apoyo social } & 3,97 & 89 & 4,18 &, 81 & $-3,03$ \\
\hline & & & & & $(631)^{* *}$ \\
\hline \multirow[t]{2}{*}{ Resolución pacífica de problemas } & 3,44 & ,99 & 3,72 &, 89 & $-3,72$ \\
\hline & & & & & $(629)^{* *}$ \\
\hline
\end{tabular}

Ahora bien, ¿explica esta exposición diferencial la menor tendencia de las chicas a participar en conductas violentas? Para comprobarlo, se ha realizado un análisis de regresión logística. El análisis de regresión logística permite pronosticar la pertenencia a un grupo a partir de diversas variables independientes. En este sentido, tiene una función similar al análisis discriminante, pero permite trabajar con datos que no cumplen los requisitos del análisis discriminante y con variables dicotómicas. En nuestro caso, nos interesa conocer cuáles de las variables estudiadas, incluyendo el sexo, tienen un peso significativo para discriminar entre sujetos de alto y bajo riesgo de 
conducta violenta. Para ello, hemos seleccionado dos grupos extremos: los de bajo riesgo violento ( $35 \%$ de la muestra, $n=225)$, conformado por aquellos que no han cometido ni una sólo conducta violenta, y los de alto riesgo $(23,8 \%$ de la muestra, $\mathrm{n}=153$ ), conformado por aquellos que han cometido tres o más conductas distintas (superior a la media).

La introducción de las variables en la regresión se hizo forzosa, lo que permite establecer el efecto conjunto de todas las variables cuando existe colinealidad. Incluimos sólo aquellas variables que han mostrado relaciones significativas con la conducta violenta en los análisis anteriores. En la Tabla 3 se recogen los principales estadísticos del modelo obtenido y las variables significativas en el mismo. El modelo obtenido es capaz de explicar el $37,7 \%$ de la varianza entre los individuos violentos y no violentos y permite clasificar correctamente al 77,6\% de los sujetos. En todo caso, no nos interesa tanto obtener un modelo predictivo como conocer qué variables de las analizadas tienen un efecto significativo independiente del resto. Los resultados muestran que sólo tres variables tienen ese efecto protector significativo: el sexo (ser chica), la supervisión familiar y la solución pacífica de problemas. Mientras que buscar apoyo social frente a los problemas, en contra de lo esperado, incrementa significativamente el riesgo de ser violento. Así pues, ser chica tiene un efecto protector que es independiente de su mayor exposición a ciertos factores protectores.

\begin{tabular}{|lccc|}
\hline Tabla 3. Regresión logística (variables en la ecuación) & & & \\
\hline \multicolumn{1}{|c}{ VARIABLES INDEPENDIENTES } & B & WALD (gl) & EXP (B) \\
Sexo (chica) &,- 908 & $6,213(1)^{*}$ &, 403 \\
Solución pacífica & $-1,283$ & $27,51(1)^{* *}$ &, 277 \\
Supervisión familiar &,- 390 & $5,90(1)^{*}$ &, 677 \\
Busca apoyo social &, 627 & $6,61(1)^{*}$ & 1,87 \\
2 log. de la verosimilitud & & 247,850 & \\
R cuadrado de Cox y Snell & &, 377 & \\
Porcentaje clasificado correctamente & $77,6 \%: 84,2 \%$ no violentos, $68,1 \%$ violentos \\
\hline * $\mathrm{p} \leq .05 * * \mathrm{p} \leq .01$ & & &
\end{tabular}


Finalmente, y de acuerdo con el último objetivo del trabajo, se pretende aclarar si las diferencias respecto a conducta violenta entre chicos y chicas se deben, al menos en parte, a un efecto diferencial de los factores protectores. Para determinar qué factores protectores tienen una relación significativa con el índice de violencia en chicos y en chicas, y analizar si existen realmente diferencias en el efecto de estas variables, se han hallado correlaciones bivariadas entre las variables estudiadas con el índice de violencia.

Tabla 4. Efecto diferencial de los factores de protección según sexo

\begin{tabular}{|c|c|c|}
\hline \multirow[t]{2}{*}{ Factores protectores } & \multicolumn{2}{|c|}{ Índice violencia } \\
\hline & Chicos & Chicas \\
\hline Vinculo escolar &,$- 200 *$ &,$- 132 *$ \\
\hline Participación significativa en la escuela &,$- 278 * *$ & n.s. \\
\hline Trato justo de los profesores &,$- 323 * *$ &,- 177 \\
\hline Participación significativa en casa &,$- 265 * *$ &,$- 202 * *$ \\
\hline Expectativas familiares &,$- 188 * *$ & n.s. \\
\hline Supervisión familiar &,$- 230 * *$ & n.s. \\
\hline Relación con la madre &,$- 256 * *$ &,$- 267 * *$ \\
\hline Relación con el padre &,$- 325 * *$ &,$- 204 * *$ \\
\hline Apoyo de amigos & n.s. &, $131 *$ \\
\hline Amigos prosociales &,$- 285 * *$ &,- 336 \\
\hline Empatía &,$- 189 * *$ & n.s. \\
\hline Autoeficacia &,$- 201 * *$ & n.s. \\
\hline Autoconocimiento &,$- 134^{*}$ & n.s. \\
\hline Objetivos de futuro &,$- 214 * *$ &,$- 285 * *$ \\
\hline Apoyo social &,$- 166^{* *}$ & n.s. \\
\hline Resolución pacífica de problemas &,$- 448 * *$ &,$- 348 * *$ \\
\hline
\end{tabular}

Debemos destacar, en primer lugar, que existen más factores que tienen un efecto protector en los chicos que en las chicas y que la fuerza de la relación es, en general, también mayor en los chicos. 
Entre los factores que tienen un efecto protector importante en los chicos, pero no en las chicas, cabe destacar la participación significativa en la vida escolar, la supervisión familiar y tener una buena relación con el padre. Tener una forma pacífica de resolver los problemas, hablando o escribiendo, es el factor con una relación más fuerte tanto en chicos como en chicas, pero claramente mayor en los chicos,

Los resultados indican que sólo dos factores tienen un efecto mayor en las chicas: tener amigos pro sociales, que no se meten en problemas, y tener objetivos de futuro.

\section{Conclusiones y discusión}

En conjunto, los datos analizados aportan información interesante pero también controvertida sobre los factores protectores frente a la conducta violenta en chicas y chicos

Los chicos y las chicas presentan más semejanzas que diferencias en sus patrones de conducta antisocial. Como muestran los resultados, y en consonancia con las previsiones de estudios anteriores sobre este tema (Bartolomé, 2001), existe actualmente una notable equiparación de las chicas respecto a los chicos, sobre todo en conductas de consumo y no violentas. Una posible explicación de las semejanzas entre chicos y chicas la encontramos dentro de la perspectiva de la Criminología Evolutiva, que plantea que implicarse en conductas antisociales alguna vez es algo propio, incluso normativo, de la adolescencia (Moffit, 1993 y 2006). Por tanto, en nuestra opinión, no es que las chicas estén adoptando conductas propiamente masculinas, sino que comparten con los chicos una forma de comportarse normativa en los jóvenes de nuestra sociedad, especialmente en contextos de ocio y con los iguales, que incluye la participación en conductas antisociales (Bartolomé y Rechea, 2005).

A pesar de la existencia de más semejanzas que diferencias entre ambos grupos en cuanto a la conducta antisocial, los datos muestran que hay menos chicas que participan en conductas violentas. Además, los chicos presentan una mayor incidencia y variedad de conductas violentas, lo que estaría relacionado con una mayor probabilidad de continuar una carrera delictiva (Tolan y Gorman-Smith, 1998). Estos resultados son acordes con los señalados por la literatura científica en este campo (Chesney-Lind, 2004; Loeber y Stouthamer-Loeber, 1998; Surgeon General, 2000; Weiler, 1999). 
Ahora bien, ¿Cómo explicar estas diferencias? Respecto a la propuesta teórica de una exposición diferencial a los mismos factores de protección, nuestros datos muestran que las chicas están más expuestas a ciertos factores de protección que los chicos. Fundamentalmente, están más supervisadas por sus padres y muestran vínculos más fuertes con amigos pro sociales. Además, tienen mayor interés en seguir estudiando. Esto es lo esperado de acuerdo con las teorías de control social (Alarid, Burton y Cullen, 2000). Sin embargo, nuestros análisis indican que las diferencias respecto a la conducta violenta en chicos y chicas no se explican únicamente por esta exposición diferencial, ya que el análisis de regresión muestra que el sexo constituye, en sí misma, una variable que pronostica de forma significativa la conducta violenta. Es decir, los efectos de riesgo y protección de la variables sexo van más allá del efecto producido por el resto de variables analizadas en este trabajo; estos resultados son acordes con los obtenidos en estudios similares (Fitzgerald, 2003). Una posible explicación es que las diferencias en violencia puedan estar relacionadas con la existencia de diferencias tempranas bio-sociales que hacen que las chicas sean menos activas y agresivas, y que muestren una mayor tendencia a desarrollar problemas de internalización como respuesta a ciertos riesgos (Keenan y Shaw, 1997; Leadbeater, Kupermine, Blatt y Hertzog., 1999), diferencias que se hacen más manifiestas con la socialización.

Por último, y respecto a si existe un efecto diferencial de los mismos factores de riesgo y protección en función del sexo, la conclusión a la que podemos llegar es que sí existe un efecto diferente, pero, al contrario de lo que sería lógico pensar, hay más factores que tienen efecto protector sobre los chicos; incluso las relaciones con el padre tienen un efecto mayor en los chicos, lo que va en contra de de los resultados de otras investigaciones (Alarid et al., 2000). En general, hay cierto acuerdo en considerar que el efecto de los factores de riesgo es mayor en los chicos (Fagan, Van Horn, Hawkins y Arthur, 2007). Pero si más factores de los estudiados tienen un efecto protector, ¿por qué no están finalmente los chicos mas protegidos frente a la conducta antisocial? Pensamos que estos resultados pueden ser consecuencia del escaso conocimiento sobre la conducta violenta en las chicas que conlleva que los factores que les protegen a ellas todavía no estén bien identificados. La literatura se está haciendo eco de que existen tantas contradicciones en los resultados obtenidos sobre la cuestión género/delincuencia, 
que es necesario mejorar el conocimiento sobre las chicas, pero también cambiar cómo se plantean las investigaciones (Bartolomé, 2001; Chapple et al., 2005; Weiler, 1999). A pesar de ello, sí merece la pena destacar un importante efecto en las chicas de los factores de protección relacionados con tener objetivos de futuro y tener planes de seguir estudiando. Estas podrían ser cuestiones a tener especialmente en cuenta a la hora de diseñar programas de intervención y prevención dirigidos a la conducta violenta en las chicas. En cambio, la búsqueda de apoyo social ante los problemas, una estrategia más habitual en mujeres, no les protege de la conducta antisocial en estas edades, lo que puede estar relacionado con que el apoyo que buscan es el de los amigos o pareja, con los que comparten estilo de comportamiento en gran medida. 


\section{Referencias}

Alarid, L. F., Burton, V. S. y Cullen, F. T. (2000). Gender and crime among felony offenders: assessing the generality of social control and differential association theories. Journal of Research in Crime and Delinquency, 37 (2), 171-199.

Bartolomé, R. (2001). Delincuencia juvenil femenina. Tesis Doctoral UCLM no publicada.

Bartolomé, R. y Rechea C. (2005).Violencia y conducta antisocial. En: Libro de ponencias del Congreso ser adolescentes hoy, 213-224. Madrid: FAD.

Belknap, J. (1996). The invisible woman. Belmont: Wadsworth P. C.

Belknap, J. and Holsinger, K. (1998). An overview of delinquent girls: How theory and practice have failed and the need for innovative changes. En R. T. Zaplin, Female offenders: Critical perspectives and effective interventions. Gaithersburg: Aspen Publishers.

Bernard, B (999). Application of Resilence: possibilities and promise. In M. D. Glantz and J.L. Johnson (Eds.) Resilience and Development. Positive Life Adaptations. N.Y.: Kluwer Academic/Plenum Publishers. Pp. 269-279

California Healthy Kids Survey, (2002). Disponible en http://www.cde.ca.gov/ls/yd/re/chks.asp

Chapple, C. L., McQuillan, J. A. and Berdahl, T. A. (2005). Gender, social bonds and delinquency: a comparison of boys' and girls'. Models .Social Science Research 34, 357-383.

Chesney-lind, M. (1997). The female offender. Thousand Oaks: Sage Publications.

Chesney-Lind, M. (2004). Girls and violence: is the gender gap closing? Applied Research Forum. Disponible en http://www.vawnet.org/DomesticViolence/Research/VAWnetDocs/AR_GirlsVi olence.pdf. Consultado en Mayo 2007.

Constantine, N. Benard, B. and Díaz, M. (1999). Measuring protective factors and resilience traits in youth: The healthy kids resilience assessment. Comunicación presentada en la Seventh Annual Meeting of the Society for Prevention Research. New Orleans, LA, Junio, 1999.

Fagan, A. A., Van Horn, M. L., Hawkins, D. y Arthur, M. W. (2007). Gender similarities and differences in the association between risk and protective factors and self-reported serious delinquency. Prevention Science 8 (2), 115-124.

Fitzgerald, R. (2003). An examination of sex differences in delinquency. Crime and Justice research paper series. Disponible en http://ww4.pssp.gc.ca/en/library/publications/youth/gender/85-561-mie2003001.pdf.

Consultado en mayo de 2007.

.Heimer, K., De Coster, S. (1999). The gendering of violent delinquency. Criminology 37, 277-317.

Keenan, K. and Shaw, D. S. (1997). Developmental and social influences on young girls' early problem behavior. Psychological Bulletin, 121, 95-113.

Leadbeater, B. J., Kupermine, G. P., Blatt, S. J. and Hertzog, C. (1999). A multivariate model of gender differences in adolescents. Internalizing and externalizing problems. Developmental Psychology, 35 (5), 1268-1282.

Lipsey, M.W. and Derzon, J.H. (1998). Predictors of Violent or Serious Delinquency in Adolescense and Early Adulhood. A Synthesis of Longitudinal Research. In R. 
Loeber y D. P. Farrington (Eds.). Serious ans Violent Juvenile Offenders. Risk Factors and Successful Interventions (Pp. 86-104). USA: Sage Publications.

Loeber, R. and Stouthamer-Loeber, M. (1998). Development of juvenile aggression and violence. Some common misconceptions and controversies. American Psychologist, 53, 242-259.

Lösel, F. and Bender, D. (2003). Protective Factors and Resilience. In D.P. Farrington and J.W. Coid (Eds.) Early Prevention of Adult Antisocial Behavior (Pp. 130204). UK: Cambridge University Press..

Moffitt, T. E. (1993). Adolescence-limited and life-course-persistent antisocial behavior: a developmental Taxonomy. Psychological Review, vol. 100 (4), 674701. M

Moffitt, T.E. (2006). Life-course persistent versus adolescence-limited antisocial behavior. In D. Cicchetti \& D. Cohen (Eds.). Developmental Psychopathology. (Pp. 570-598) NY: Wiley

Pollock, J.M. (1999). Criminal Women. Cincinnati: Anderson Publishing Co.

Rechea, C., Barberet, R. y Montañés, J. (1995). La delincuencia Juvenil en España: Autoinforme de los Jóvenes. Madrid: Ministerio de Justicia e Interior.

Reppucci N. D., Fried, S. and Schmidt, M.G. (2002). Youth Violence: Risk and Protective Factors. En R.P. Corrado, R. Roesch, S.D. Hart and J.K. Gierowski (Eds.) Multi-Problem violent youth. A foundation for comparative research on needs, interventions and outcomes. Amsterdam: IOS Press.

Rutter, M., Giller, H. y Hagell, A. (2000): La conducta antisocial de los jóvenes. Madrid: Cambridge University Press

Steffensmeier, D., Allan, E., (1996). Gender and crime: toward a gendered theory of female offending. Annual Review of Sociology 22, 459-487..

Surgeon General (2000): Youth violence, disponible en http://www.surgenogeneral.gov.

Tolan P.H. and Gorman-Smith D. (1998). Development on Serious and Violent Offending Careers. En R. Loeber and D. P. Farrington (Eds.). Serious and Violent Juvenile Offenders. Risk Factors and Successful Interventions (Pp. 6885). USA: Sage Publications.

Weiler, J. (1999): Girls and violence. New York: ERIC Clearinghouse on Urban Education. ERIC document No ED 430069. 
Correspondencia: Raquel Bartolomé. Centro de Investigación en Criminología. Edificio Benjamín Palencia. Campus Universitario. 02071 Albacete. E-mail: Raquel.Bartolome@uclm.es

Agradecimientos: Los datos utilizados en este trabajo pertenecen a un proyecto más amplio sobre Factores de protección en el consumo de alcohol y otras conductas problemáticas en adolescentes financiado por la CICYT (BS 02002-03921). Agradecemos su colaboración a la Delegación de Educación de Albacete y a los orientadores, profesores y alumnos de los centros en que se pasó la encuesta.

\section{Sobre los autores}

Raquel Bartolomé es Doctora en Psicología e investigadora del CIC de la UCLM Marta Montañés es Licenciada en Psicología y actualmente está realizando su doctorado en el CIC.

Cristina Rechea es Catedrática de Psicología Básica y Directora del CIC de la UCLM. Juan Montañés es Catedrático de EU de de Psicología Evolutiva y Director del Departamento de Psicología de la UCLM.

Todos ellos tienen como área de estudio la conducta antisocial y delictiva de menores y jóvenes. 